\title{
Daya Bay Results
}

\author{
Viktor Pěč ${ }^{* \dagger}$ \\ Charles University, Prague \\ E-mail: pec@ipnp.troja.mff.cuni.cz
}

The Daya Bay experiment was designed to measure neutrino mixing parameter parameter $\theta_{13}$ with unprecedented precision by employing a relative rate measurement of electron antineutrinos at short and long distance from nuclear reactors. Updated analysis of spectral and rate differences at different distances based on 1260 days of data yielded $\sin ^{2} 2 \theta_{13}=0.0841 \pm$ 0.0027 (stat.) \pm 0.0019 (syst.), together with a measurement of the effective squared-mass difference $\Delta m_{\mathrm{ee}}^{2}=(2.50 \pm 0.06$ (stat.) \pm 0.06 (syst. $\left.)\right) \times 10^{-3} \mathrm{eV}^{2}$. Latest results of independent analysis of events with neutron capture on hydrogen, of the measurement of absolute reactor antineutrino flux and spectrum, and of the search for mixing of sterile neutrinos are also reported. The experiment is expected to continue running till 2020.

Neutrino Oscillation Workshop

4 - 11 September, 2016

Otranto (Lecce, Italy)

\footnotetext{
* Speaker.

${ }^{\dagger}$ for the Daya Bay Collaboration
} 


\section{Introduction}

The Daya Bay Experiment detects reactor antineutrinos $\bar{v}_{\mathrm{e}}$ from 6 reactor cores of nuclear power complex in China. The reactor cores are grouped by two into three nuclear power plants. One power plant is at the Daya Bay site and the other two are at Ling Ao site.

The experiment determines the oscillation parameters $\theta_{13}$ and $\Delta m_{e e}^{2}$ from comparison of $\bar{v}_{\mathrm{e}}$ rate and energy spectrum at different baselines from the reactor cores. In total, 8 antineutrino detectors are installed in three underground experimental halls. Two experimental halls, instrumented with 2 detectors each, are located near the reactor cores, at distances about $350 \mathrm{~m}$ and $500 \mathrm{~m}$ from the Daya Bay and Ling Ao power plants, respectively. The third experimental hall, which hosts 4 detectors, is located at distances $\sim 2 \mathrm{~km}$ and $1.6 \mathrm{~km}$ from the cores. The experiment started taking data with only 6 operational detectors installed in 2011. The full 8 detector run started in 2012 after summer maintenance and installation break.

The reactor antineutrinos are detected in the inverse $\beta$ decay (IBD) interactions on hydrogen, $\bar{v}_{\mathrm{e}}+\mathrm{p} \rightarrow \mathrm{e}^{+}+\mathrm{n}$. The events are reconstructed from two consecutive signals. The prompt signal comes from the kinetic energy of the positron and from gamma photons of its annihilation. The delayed signal comes from the neutron capture on a nucleus. In case of Daya Bay it is dominantly gadolinium which emits cascade gamma photons with total energy of $8 \mathrm{MeV}$ (nGd events), or hydrogen which emits 2-MeV gamma (nH events).

The Daya Bay antineutrino detectors are $5 \mathrm{~m}$ by $5 \mathrm{~m}$ cylindrical stainless steel vessels which consist of three nested cylindrical zones partitioned with two cylindrical acrylic tanks. The innermost region is filled with gadolinium-loaded organic liquid scintillator and serves as the antineutrino target for the main analyses of nGd events.

The middle layer is filled with plain liquid scintillator. The outer most layer is filled with transparent mineral oil. The light produced in the scintillator is collected by 1928 -inch photomultiplier tubes. The two scintillator layers weigh about 20 ton each. More detailed information about the antineutrino detectors can be found in [1].

The detectors at each experimental hall are placed inside water pools which are divided into two optically separated regions, each of which is instrumented with PMTs and works as an independent Cherenkov detector of cosmic ray muons. The pools are covered with multi-layered resistive plate chambers. The muon detection system is extensively described in [2].

\section{Results}

Oscillation results with sample of nGd events based on data from a period of 1230 days were recently released in [3]. The total rate of $\bar{v}_{\mathrm{e}}$ and the energy spectrum of the prompt signal was compared between the experimental halls. Deficit in the far hall was fitted with the oscillation formula $P_{\bar{v}_{\mathrm{e}} \rightarrow \bar{v}_{\mathrm{e}}} \simeq 1-\sin ^{2} 2 \theta_{13} \sin ^{2} \Delta_{\mathrm{ee}}-\cos ^{4} \theta_{13} \sin ^{2} 2 \theta_{12} \sin ^{2} \Delta_{21}$, with $\Delta_{i j}=\frac{\Delta m_{i j}^{2} L}{4 E}$. The analysis yielded $\sin ^{2} 2 \theta_{13}=0.0841 \pm 0.0027$ (stat.) \pm 0.0019 (syst.) and $\Delta m_{\mathrm{ee}}^{2}=(2.50 \pm 0.06$ (stat.) \pm 0.06 (syst.) $) \times$ $10^{-3} \mathrm{eV}^{2}$ with $\chi^{2} / \mathrm{ndf}=232.6 / 263$. The Figure 1 a shows measured $\bar{v}_{\mathrm{e}}$ survival probability with respect to effective distance.

Independent oscillation analysis based on $\mathrm{nH}$ events has recently been updated in [4]. The measurement which compared relative IBD rates in the Daya Bay near and far detectors, and based 


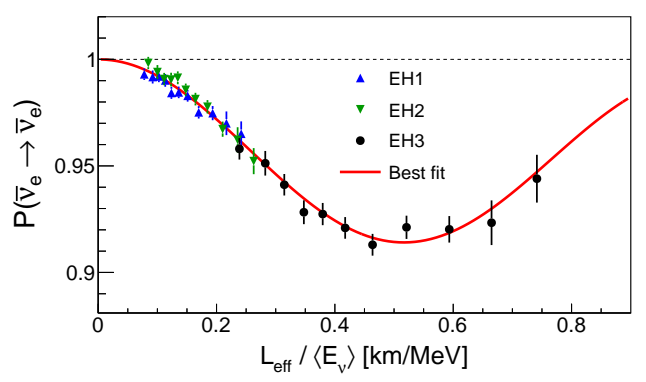

(a)

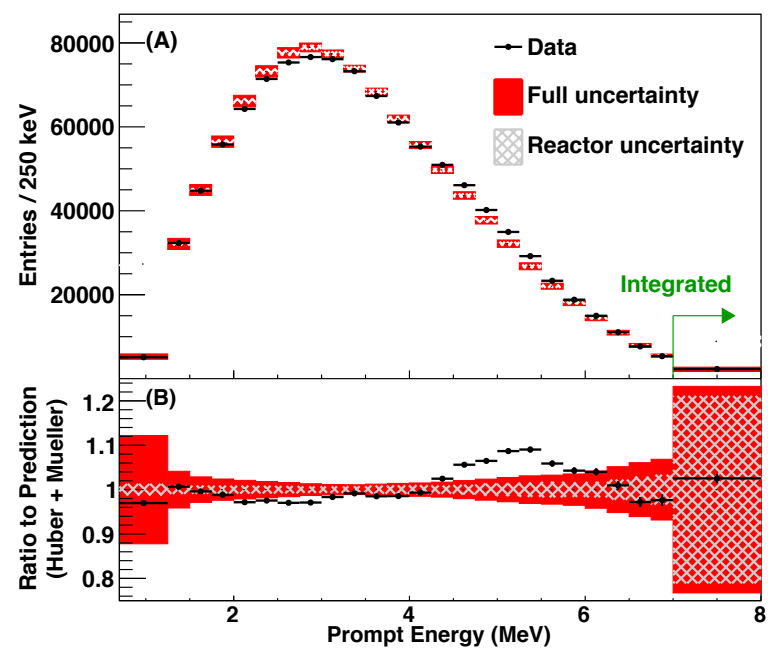

(b)

Figure 1: (a) Measured survival probability of $\bar{v}_{\mathrm{e}}$ with respect to effective distance from the source obtained from distortions of $\bar{v}_{\mathrm{e}}$ energy spectrum in each hall. (b) Spectrum of prompt energies of $\bar{v}_{\mathrm{e}}$ interactions in near detectors, compared to the Huber+Mueller prediction.

on data set from period of 621 days, yielded currently the 3rd best measurement of the mixing parameter $\sin ^{2} 2 \theta_{13}=0.071 \pm 0.011$ with $\chi^{2} / \mathrm{ndf}=6.3 / 6$. This measurement is largely independent from the result of the analysis of the nGd events due to different sample and largely distinct systematic uncertainties. Correlation coefficient of the total systematic uncertainties of the two analyses is 0.02 .

High statistics of antineutrino events gives opportunity to precisely measure the absolute reactor antineutrino flux and shape of the energy spectrum. A recently released improved measurement [5] is based on exposure of 621 days and makes use of over 1.2 million $\bar{v}_{\mathrm{e}}$. The measured IBD yield is $Y=(1.55 \pm 0.03) \times 10^{-18} \mathrm{~cm}^{2} / \mathrm{GW}_{\text {th }} /$ day. The ratio of the yield to the expectation from the Huber+Mueller model [6, 7] is $R=0.946 \pm 0.020$ (exp.), which is consistent with measurements of the past short baseline reactor experiments.

The spectrum of reactor antineutrinos exhibit inconsistency with existing models. There is an excess of events in the energy range $4-6 \mathrm{MeV}$ as can be seen in Figure 1b. The excess is correlated with the reactor power and resembles all features of IBD events. No similar distortion is observed in $\beta$ spectrum of ${ }^{12} \mathrm{~B}$ disfavoring detector related effects.

An update on possible existence of light sterile neutrinos and their mixing with standard neutrinos probed with Daya Bay was published in [8]. No difference is observed between the measured spectrum of prompt energies and the prediction based on the 3-neutrino oscillation formula. With data collected over the 621 days most stringent limits on the sterile neutrino mixing were set in the region $\left|\Delta m_{41}\right| \lesssim 0.2 \mathrm{eV}^{2}$.

The measurement of $\bar{v}_{\mathrm{e}}$ disappearance by Daya Bay was combined with $\bar{v}_{\mathrm{e}}$ disappearance measurements of Bugey-3 [9] and with $v_{\mu}$ disappearance measurement of MINOS [10]. The reported findings [11] probe regions of mixing parameters allowed by observation of $\bar{v}_{\mathrm{e}}$ by the LSND [12] and MiniBooNE [13] experiments. Part of the parameter space $\Delta m_{41}^{2},\left|U_{\mathrm{e} 4}\right|\left|U_{\mu 4}\right|$ is excluded for 
$\Delta m_{41}^{2}<0.8 \mathrm{eV}^{2}$.

\section{Outlook}

The Daya Bay Experiment is expected to continue running till 2020. Systematic uncertainties related to the detector performance will be further reduced through the installation of a FADC readout system and through special calibration campaign. Increased statistics and reduced systematics will aid our goal to reach precision in $\sin ^{2} 2 \theta_{13}$ and $\Delta m_{\mathrm{ee}}^{2}$ to better than $3 \%$.

\section{Acknowledgments}

The author was supported by the Ministry of Education, Youth and Sports of the Czech Republic.

\section{References}

[1] F.P. An et al. (Daya Bay Collaboration), The Detector System of The Daya Bay Reactor Neutrino Experiment, Nucl. Instrum. Meth. A 811 (2016) 133 [arXiv:1508.03943 [physics.ins-det]].

[2] F.P. An et al. (Daya Bay Collaboration), The muon system of the Daya Bay Reactor antineutrino experiment, Nucl. Instrum. Meth. A 773 (2015) 8 [arXiv:1407.0275 [physics.ins-det]].

[3] F. P. An et al. [Daya Bay Collaboration], Measurement of electron antineutrino oscillation based on 1230 days of operation of the Daya Bay experiment, arXiv:1610.04802 [hep-ex].

[4] F.P. An et al. (Daya Bay Collaboration), New measurement of $\theta_{13}$ via neutron capture on hydrogen at Daya Bay, Phys. Rev. D 93 (2016), 072011 [arXiv:1603.03549 [hep-ex]].

[5] F. P. An et al. [Daya Bay Collaboration], Improved Measurement of the Reactor Antineutrino Flux and Spectrum at Daya Bay, arXiv:1607.05378 [hep-ex].

[6] P. Huber, On the determination of anti-neutrino spectra from nuclear reactors, Phys. Rev. C 84 (2011) 024617 [arXiv:1106.0687 [hep-ph]].

[7] T. A. Mueller et al., Improved Predictions of Reactor Antineutrino Spectra, Phys. Rev. C 83 (2011) 054615 [arXiv:1101.2663 [hep-ex]].

[8] F.P. An et al. (Daya Bay Collaboration), Improved Search for a Light Sterile Neutrino with the Full Configuration of the Daya Bay Experiment, Phys. Rev. Lett. 117 (2016), 151802 [arXiv:1607.01174 [hep-ex]].

[9] Y. Declais et al., Search for neutrino oscillations at 15-meters, 40-meters, and 95-meters from a nuclear power reactor at Bugey, Nucl. Phys. B 434 (1995) 503.

[10] P. Adamson et al. (MINOS Collaboration), Search for Sterile Neutrinos Mixing with Muon Neutrinos in MINOS, Phys. Rev. Lett. 117 (2016), 151803 [arXiv:1607.01176 [hep-ex]].

[11] P. Adamson et al. (Daya Bay and MINOS Collaborations), Limits on Active to Sterile Neutrino Oscillations from Disappearance Searches in the MINOS, Daya Bay, and Bugey-3 Experiments, Phys. Rev. Lett. 117 (2016), 151801 [arXiv:1607.01177 [hep-ex]].

[12] A. Aguilar-Arevalo et al. (LSND Collaboration), Evidence for neutrino oscillations from the observation of anti-neutrino(electron) appearance in a anti-neutrino(muon) beam, Phys. Rev. D 64 (2001) 112007 [hep-ex/0104049].

[13] A. A. Aguilar-Arevalo et al. (MiniBooNE Collaboration), Improved Search for $\bar{v}_{\mu} \rightarrow \bar{v}_{e}$ Oscillations in the MiniBooNE Experiment, Phys. Rev. Lett. 110 (2013) 161801 [arXiv:1303.2588 [hep-ex]]. 